\title{
Intravascular leiomyomatosis: unusual variant of leiomyoma
}

\author{
Sameer Umranikar • Aarti Umranikar • \\ Benjamin Byrne • Adam Moors
}

Received: 9 June 2008 / Accepted: 1 August 2008 / Published online: 27 August 2008

(C) Springer-Verlag 2008

\begin{abstract}
Leiomyomas (fibroid) arise from the smooth muscle of the myometrium and are benign in nature. Intavascular leiomyomatosis is an unusual and rare condition where the fibroid grows into the pelvic veins and inferior vena cava and sometimes extends into the heart chambers. We present a case where a woman presented to us with multiple fibroids and underwent an abdominal hysterectomy. The diagnosis was not suspected before the surgery but diagnosed on histopathology. The patient underwent further surgery to remove the fibroids from the pelvic veins and the inferior vena cava. This condition has been reported in only few case reports around the world. In view of the rarity of this condition, the diagnosis can be easily missed. We suggest an increased vigilance in women who have large fibroids with unusual features in symptoms or during surgery. Pre-surgical imaging will help to make diagnosis, and good surgical outcomes can be achieved by a multidisciplinary surgical approach.
\end{abstract}

Keywords Fibroid - Leiomyomatosis · Hysterectomy · Intravascular $\cdot$ Estrogen receptors $\cdot$ Progesterone receptors

S. Umranikar $(\bowtie) \cdot A$. Umranikar $\cdot$ A. Moors

Obstetrics and Gynaecology, Princess Anne Hospital,

Hampshire, UK

e-mail: drsaumranikar@hotmail.com

S. Umranikar

Obstetrics and Gynaecology,

Basingstoke and North Hamsphire Foundation Trust Hospital,

Basingstoke, UK

B. Byrne

Cardiothoracic, Southampton General Hospital,

Southampton, UK

\section{Introduction}

Intravascular leiomyomatosis (IVL) is a rare and benign variant of leiomyoma. It is characterised by smooth muscle proliferation in pelvic veins. They have a malignant characteristic by which they grow into pelvic veins, and the inferior vena cava (IVC) sometimes extends into the chambers of the heart and pulmonary vasculature. This condition was first described by Birch-Hirschfeld [1] in 1896. We describe a case of intravascular leiomyomatosis in a 39 -year-old woman.

\section{Case report}

A 39-year-old woman was referred to our hospital with symptoms of menorrhagia and a pelvic mass. She had been suffering from heavy periods for 6 months before her presentation and noticed abdominal distension during that period. On examination, she was found to have an enlarged uterus equivalent to $18-20$ weeks gestation, which was confirmed on pelvic examination.

Ultrasound examination revealed an enlarged uterus with multiple fibroids with no unusual features. Following consultation and counselling, she underwent a total abdominal hysterectomy with conservation of ovaries. The diagnosis of intravascular leiomyomatosis was not suspected before her surgery.

At surgery, the uterus was noted to be equivalent to a gestation size of 20 weeks with restricted mobility. The whole of the pelvis was occupied by fibroids with extension of the fibroids into the right broad ligament and towards the right infundibulo-pelvic ligaments. The fibroids were also noted to be extending towards the cervix and vagina. Both the ovaries and fallopian tubes were normal. A total 


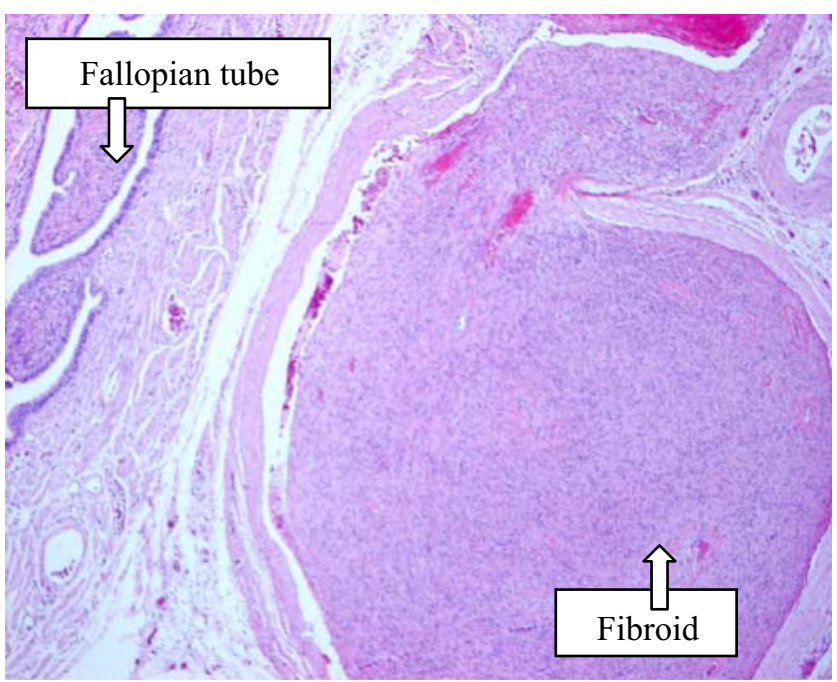

Fig. 1 Spindle-shaped cells of the leiomyoma next to the fallopian tube

abdominal hysterectomy was carried out. A right salpingooopherectomy was done at the same time as the fibroids were extending into the right infundibulopelvic and broad ligaments. A few fibroids in the broad ligament were not removed due to their proximity to the ureter.

At histology, the uterus measured $100 \times 90 \times 65 \mathrm{~mm}$, and the cervix measured $60 \times 38 \mathrm{~mm}$. The fibroids were polypoidal in nature, extending from the endometrium towards the serosal surface of the uterus, which was breached. The fibroid mainly consisted of interlacing fascicles of spindle cells with cigar-shaped nuclei. The cells were uniform and bland, and the mitotic count was 0 per ten high-power fields. A large proportion of the fibroid was within blood vessels, and it appeared to be attached to the vessel wall in places. The fibroid was also noted to be within the vessels of the fallopian tube wall, ovarian hilum and the cervical canal stroma. The histological characteristics were benign in nature.

The fibroids were tested using immunohistochemical staining and were positive for muscle markers for smooth muscle actin and desmin. The CD34 staining highlighted the predominant intravascular nature of the tumour (Figs. 1 and 2). Hence, the diagnosis of intravascular leiomyomatosis was confirmed histologically. On further testing, the tumour was found to be estrogen and progesterone receptor positive.

The patient made a very good post-operative recovery. She underwent further imaging with a magnetic resonance imaging (MRI) and computed tomography (CT) scan, which revealed the leiomyomas extending into her right common iliac veins and into the inferior vena cava just beyond the renal vessels (Figs. 3 and 4). There was no extension of the tumour noted in the cardiac chambers.

In view of the presence of estrogen and progesterone receptors, she was treated with 4 months of GnRH analogues, and a second operation was undertaken jointly with the vascular surgeons to remove the leiomyomas from the inferior vena cava and iliac vessels (Figs. 3 and 4). During the surgery, the remaining pelvic leiomyomas had significantly reduced in size probably due to her treatment with the GnRH analogue. She underwent oophorectomy of the remaining ovary. The inferior vena cava leiomyoma was easily stripped off the vessel wall (Fig. 5) and measured (Fig. 6)

The patient recovered well from her second surgery.

\section{Discussion}

Leiomyomas are benign tumours which arise from the smooth muscle cells of the myometrium. Intravascular leiomymatosis is an uncommon and rare variant of leiomyoma. It is characterised by smooth muscle proliferation in the walls of the venous system. They are benign tumours confined to the lumen of the venous system; however, they have dangerous characteristics by which they extend through the venous system extending from the pelvic veins through the inferior vena cava to the heart chambers.

This condition was first described in Germany by Birch Hirscheld [1] in 1896. It is unclear whether they form an extension from the uterine leiomyomas or their origin is primarily from the vessel wall itself. Norris and Pramley [2] studied 14 cases of intravascular leiomyomatosis and found evidence to define both the theories, namely primary growth within the vessel wall or an extension of preexisting leiomyomas. They suggested that intravascular leiomyomatosis should be distinguished from leiomyoma with vascular invasion microscopically where the vascular growth is confined to the leiomyoma.

Intravascular leiomyomatosis is usually associated with uterine leiomyomas. Nogales et al. [3], in a series of seven

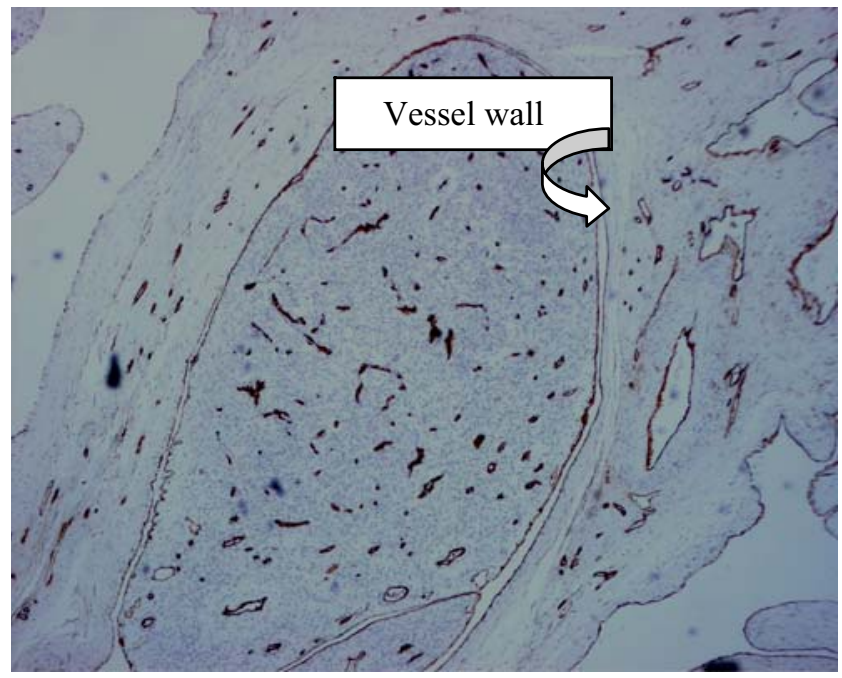

Fig. 2 CD34 staining of spindle-shaped cells in the vessel wall 


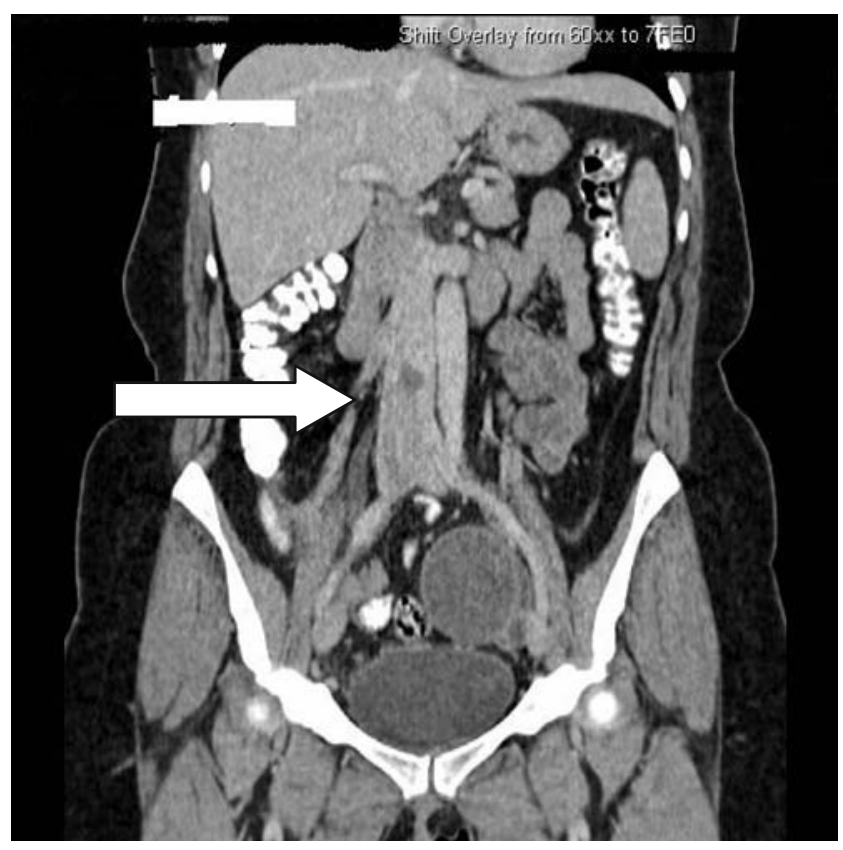

Fig. 3 CT scan image showing the filling defect in the IVC

cases of IVL, suggested that the origin of IVL occurred from a pre- existing leiomyoma. However, without any extra-uterine extension, the diagnosis can be easily overlooked. However, Kir et al. [4] reported seven cases of intravascular leiomyomatosis, looking at estrogen and progesterone expression within the vessel walls to study the histogenesis of the tumours. They found that the origin of these fibroids from the vessel wall is unlikely.

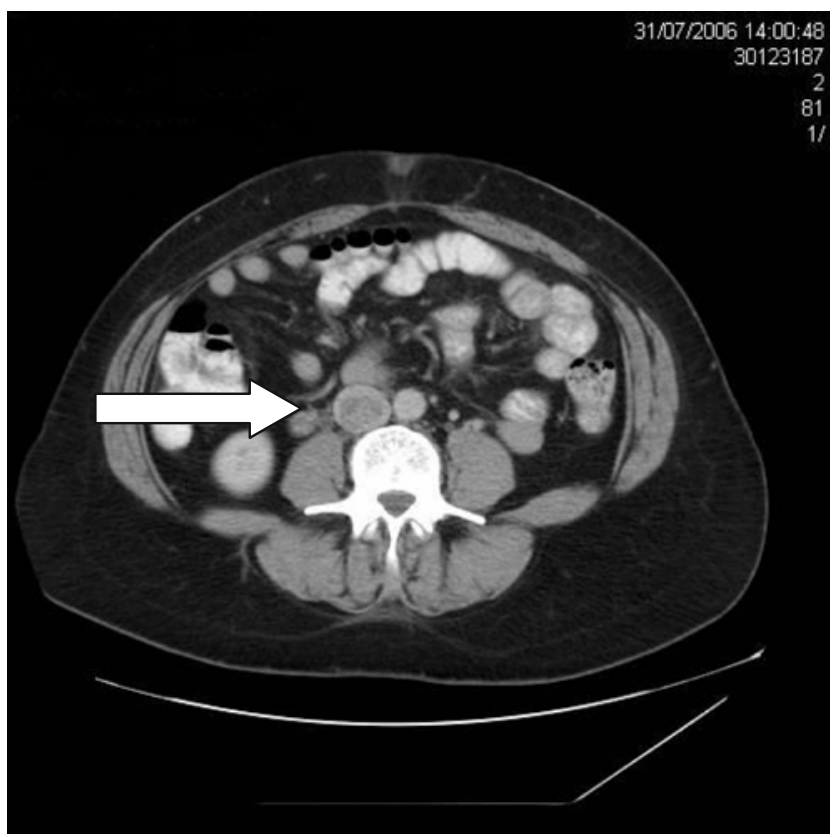

Fig. 4 CT scan imaging showing the filling defect in the IVC close to the renal veins

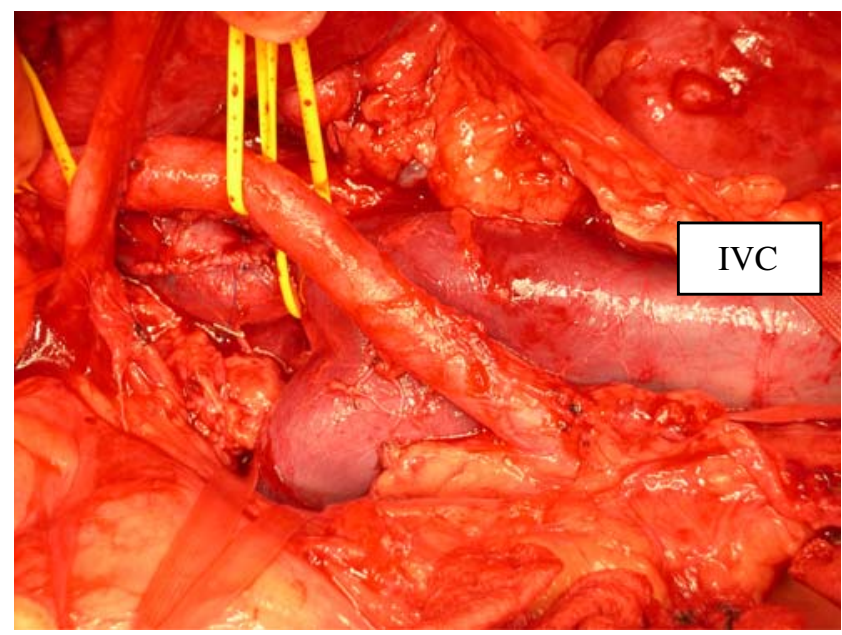

Fig. 5 Dissection of inferior vena cava to access the fibroid

Intravascular leiomyomatosis should be distinguished from other rare variants like disseminated leiomyomatosis peritonealis. Drake et al. [5] suggested that the presence of multiple masses, which contain lymphatic tissue and smooth muscle but no vascular invasion, would be more in favour of a diagnosis of disseminated leiomyomatosis peritonealis. Clement et al. [6] undertook a clinicopathological analysis in 16 cases of intravenous leiomyomatosis of the uterus with unusual histological features. They found proliferations of benign-appearing smooth muscle within the myometrial vessels and in large veins. They classified them histologically into cellular, epitheloid, lipoleiomyomatosis, myxoid and with endometrial components.

Presentation of this condition can be very varied. Noedir et al. [7] suggested that patients can be completely asymptomatic or present with symptoms related to the site of the tumours. Very commonly, this condition will co-exist with uterine leiomyomas as was seen in our case. However,

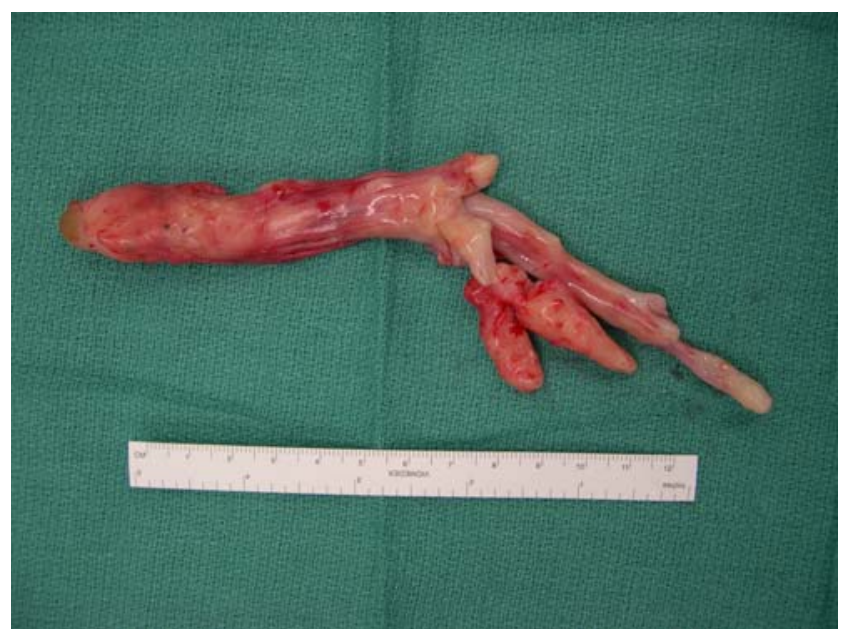

Fig. 6 The intravascular leiomyoma removed from the inferior vena cava 
tumours extending into the heart can lead to mechanical obstruction within the cardiac chambers with symptoms and signs of right-sided cardiac failure. This condition can be easily overlooked and found co-incidentally during surgery.

Lam et al. [8] reviewed 68 cases with intracardiac leiomyomatosis reported from 1900 to 2003 . They found congestive cardiac failure in 45 patients, venous obstruction in four patients and a single death from one case. They found extension of the fibroid into the right atrium in 31 cases, right ventricle in 31 cases and in the pulmonary vessels in six cases. Twenty-nine patients required a twostage operation for complete excision of the fibroids.

The definitive treatment for IVL would be a total hysterectomy with possible removal of ovaries and removal of the tumours from within the venous and cardiac systems. The presence of estrogen and progesterone receptor is a good prognostic sign, and treatment with anti estrogens may be effective. In our case, there was a substantial reduction in the tumour size noticed at the second operation following treatment with GnRH analogue. Tresukosol et al. [9] reported a case report where leuprolide acetate given preoperatively induced tumour regression to successfully carry out debulking surgery. Hence, a conservative approach using either GnRH analogues or anti-estrogens may be useful in selected patients, but these patients would need a careful follow-up for several years. Evans et al. [10] studied four cases with recurrent pelvic intravenous leiomyomatosis and found functioning ovarian tissue at the time of recurrence in three cases. They suggested bilateral oopherectomy would prevent recurrence of the disease.

IVL is a rare condition with little supporting literature. The diagnosis was not suspected in our patient preoperatively and confirmed only on histological examination of the uterus and adnexa. From our experience in the management of this case, we suggest that intravascular leiomyomatosis should be suspected during surgery where the fibroids seem to extend beyond the uterus into the broad ligaments or show extensions into vascular systems. These cases can be a surgical challenge as they extend beyond the uterus and into the pelvic side walls in close proximity to the ureters. The presence of tumour extension within the venous system would be difficult to define intraoperatively unless the patient has undergone some pre-operative imaging. Thus, a lot of these patients may need a twostage procedure for complete clearance of the fibroids. There should be a clinical suspicion in patients who have large fibroid uterus with unusual features or extensions into the pelvic side walls. The condition should also be suspected if there are other cardiac or venous compression symptoms. Extension into the cardiac and venous system can be potentially life threatening, and these patients would need to undergo further surgery to remove the fibroids.
Intravascular leiomyomatosis can be diagnosed using CT scan or MRI, whereby surgery can be planned as a singlestage procedure with an aim to remove the tumour from the pelvis and its extensions into the venous systems. Thus, a multidisciplinary approach to these cases can have a favourable outcome. However, as these fibroids have estrogen and progesterone receptors, there maybe a role to treat these patients with GnRH analogues if all of the tumour cannot be removed at initial surgery.

\section{Conclusion}

Fibroids are a common condition affecting many women; however, few clinicians may come across such a rare and unusual variant of fibroid. This case highlights the importance of pre-surgical imaging for clinically large fibroids with unusual features and symptoms. The diagnosis can be easily missed pre-operatively, and this can make the surgery very difficult and risky to the patient.

This case report highlights the need for an increased awareness of this condition. Good surgical outcomes can be achieved in treating these patients through a multidisciplinary approach.

\section{References}

1. Birch-Hirschfeld FV (1896) Lehrbuch der PathologischenAnatomie, 5th edn. Leipzig, Germany, F Wogel, p 226

2. Norris HJ, Pramley T (1975) Mesenchymal tumours of the uterus. V. Intravascular leiomyomatosis. A clinical and pathologic study of 14 cases. Cancer 36(6):2164-2178, Dec

3. Nogales FF, Navarro N, Martinez de Victoria JM, Contreras F, Redondo C, Herraiz MA, Seco MA, Velasco A (1987) Uterine intravascular leiomyomatosis: an update and report of seven cases. Int J Gynecol Pathol 6(4):331-339

4. Kir G, Kir M, Gurbuz A, Karateke A, Aker F (2004) Estrogen and progesterone expression of vessel walls with intravascular leiomyomatosis; discussion of histogenesis. Eur J Gynaecol Oncol 25(3):362-366

5. Drake A, Dhundee J, Buckley CH, Woolas R (2001) Disseminated leiomyomatosis peritonealis in association with oestrogen secreting ovarian fibrothecoma. BJOG 108:661-664, (June)

6. Clement PB, Young RH, Scully RE (1988) Intravenous leiomyomatosis of the uterus. A clinicopathological analysis of 16 cases with unusual histologic features. Am J Surg pathol 12(12):932-945, (Dec)

7. Stolf Noedir AG, dos Santos GG, Haddad VLS (1999) Unusual abdominal tumours with intracardiac extension. Two cases with successful surgical resection. Rev Hosp Clin 54(5):159-164

8. Lam PM, Lo KWK, Yu MY, Wong WS, Lau JYW, Arifi AA, Cheung TH (2004) Intravascular leiomyomatosis: Two cases with different routes of tumour extension. J VascSurg 39:465-469

9. Tresukosol D, Kudelka A, Malpica A, Varma GK, Edwards CL, Kavanagh JJ (1995) Obst Gynaecol 86:688-692

10. Evans At, Symmonds RE, Gaffey TA (1981) Recurrent pelvic intravenous leiomyomatosis. Obstet Gynecol 57(2):260-264, (Feb) 